\title{
Long-term effects of hydrogen sulfide on the anabolic- catabolic balance of articular cartilage in vitro
}

\author{
Á.Vela-Anero ${ }^{\mathrm{ab}}$, T.Hermida-Gómez ${ }^{\mathrm{bc}}$, L.Gato-Calvo ${ }^{\mathrm{c}}$, C.Vaamonde-García ${ }^{\mathrm{a}}$, S.Díaz- \\ Prado $^{\text {ab }}$, R.Meijide-Faílde ${ }^{\mathrm{a}}$, F.J.Blanco ${ }^{\mathrm{c}}$, E.F.Burguera ${ }^{\mathrm{bc}}$ \\ ${ }^{a}$ Grupo de Terapia Celular y Medicina Regenerativa, Departamento de Ciencias Biomédicas, Medicina y \\ Fisioterapia, Facultad de Ciencias de la Salud, Universidade da Coruña, Instituto de Investigación Biomédica \\ de A Coruña-Complejo Hospitalario Universitario de A Coruña, Servizo Galego de Saúde, A Coruña, Spain \\ ${ }^{b}$ CIBER de Bioingeniería, Biomateriales y Nanomedicina (CIBER-BBN), Grupo de Bioingieneria Tisular y \\ Terapia Celular (GBTTC), Spain \\ ${ }^{c}$ Grupo de Investigación en Reumatología, Instituto de Investigación Biomédica de A Coruña-Complejo \\ Hospitalario Universitario de A Coruña, Servizo Galego de Saúde, A Coruña, Spain
}

\begin{abstract}
Healthy cartilage maintenance relies on an equilibrium among the anabolic and catabolic processes in chondrocytes. With the onset of osteoarthritis (OA), increased interleukin (IL)-1 $\beta$ levels induce an inhibition of the synthesis of extracellular matrix (ECM) proteins, as well as an increase in proteases. This eventually leads to a predominance of the catabolic phenotype and the progressive loss of articular cartilage. Hydrogen sulfide $\left(\mathrm{H}_{2} \mathrm{~S}\right)$ is a small gaseous molecule recognized as the third endogenous gasotransmitter. When administered exogenously, it has shown anti-inflammatory and anti-catabolic properties in several in vitro and in vivo models. Here, OA cartilage disks were co-cultured in vitro with IL-1 $\beta(5 \mathrm{ng} / \mathrm{ml})$ and NaSH or GYY4137 (200 or $1000 \mu \mathrm{M}$ ) for 21 days. The ability of these two $\mathrm{H}_{2} \mathrm{~S}$-producing compounds to avoid long term extracellular matrix (ECM) destruction was evaluated. We used a glycosaminoglycan (GAG) quantification kit histology and immunohistochemistry (IHC) to evaluate matrix proteins degradation and matrix metalloproteinases (MMP) abundance. Through the GAGs quantification assay, safranin $\mathrm{O}(\mathrm{S}-\mathrm{O})$ and toluidine blue (TB) stains, and keratan/chondroitin sulfate $(\mathrm{KS} / \mathrm{ChS})$ IHCs it was shown that co-stimulation with $\mathrm{H}_{2} \mathrm{~S}$-forming reagents effectively avoided GAGs destruction. Both Masson's trichrome (MT) stain and collagen (col) type II IHC, as well as aggrecan (agg) IHC demonstrated that not only were these proteins protected but even promoted, their abundance being higher than in the basal condition. Further, stains also demonstrated that positivity in the interterritorial and intra-cellular for the different matrix components were rescued, suggesting that $\mathrm{NaSH}$ and GYY4137 might also have pro-anabolic effects. In addition, a clear protective effect against the increased MMPs levels was seen, since increased MMP3 and 13 levels were subsequently reduced with the co-stimulation with sulfide compounds. In general, GYY4137 was more effective than NaSH, and increasing the dose improved the results.
\end{abstract}

This study demonstrates that $\mathrm{H}_{2} \mathrm{~S}$ anti-catabolic effects, which had been previously proven in short-term (24$48 \mathrm{~h}$ ) in vitro cellular models, are maintained over time directly in OA cartilage tissue.

Keywords

Human articular cartilage; Osteoarthritis; Hydrogen sulfide; Catabolism; Proteoglycans 


\section{Introduction}

Osteoarthritis $(\mathrm{OA})$ is an heterogenous rheumatic disease that affects diarthrodial joints and results in pain, loss of mobility and function, and a reduction in quality of life. OA is a silent disease in the sense that symptoms appear when it is quite advanced. With OA, articular cartilage, responsible for the absorption and transference of loads to the subchondral bone, is progressively lost. Since cartilage is mostly avascular and aneural, only when the anatomy of the joint is severely deteriorated is the disease manifested, most significantly by joint pain. Thus, cartilage degradation is considered the hallmark of OA progression. Cartilage ECM is composed of a dense network of collagen (col) type II, glycosaminoglycans (GAGs) and proteoglycans, such as aggrecan (agg). Maintenance of this network relies on a balanced metabolic equilibrium in chondrocytes. Chondrocyte phenotype can change depending on the physiological or stress conditions [1] and a series of different phenotypes have been proposed, namely:

a) A chondroblastic phenotype, expressing the fetal collagen types II and III.

b) An anabolic phenotype that is able to maintain and regenerate the ECM synthesizing col II and proteoglycans.

c) A catabolic phenotype, in which the expression of proteolytic enzymes is increased in response to pro-inflammatory factors such as tumor necrosis factor (TNF)- $\alpha$, interleukin (IL)$1 \beta$ or nitric oxide $(\mathrm{NO})$.

d) An hypertrophic phenotype, which expresses type $X$ collagen and runt-related transcription factor 2 (runx2), among others, characteristic of the deep, mineralized zone of cartilage.

e) A fibroblastic de-differentiated phenotype, which expresses collagen type I and synthesizes fibro-cartilage, as a way to repair damaged tissue.

Under normal conditions, there is a balance between the anabolic and catabolic phenotypes for the proper maintenance of adult hyaline cartilage. However, with the development of OA, the catabolic phenotype starts to predominate leading to an imbalance between synthesis and degradation of the cartilage matrix, in addition to an increase in the hypertrophic and de-differentiated phenotypes, in an attempt to repair OA cartilage damage [2].

Increased catabolism in $\mathrm{OA}$ is the result of unnormally elevated levels of metalloproteases. These are synthesized by chondrocytes in response to inflammatory stimuli, including IL-1 $\beta$, TNF- $\alpha$ and NO. One of the most relevant metalloprotease families in OA is that of the matrix metalloproteinases (MMPs). Among these, collagenase 3 (MMP13) is the most relevant enzyme in terms of col II degradation. MMP13, is regulated by stress in general, and inflammation in particular, and it appears to play a predominant role in the early stages of OA, suggesting that its inhibition might prevent intensification of disease [3]. Additionally, stromelysin 1 (MMP3) activity has been specifically linked to proteoglycan loss [4], but it can, in addition, degrade not only col II and agg, but also other non-collagenous matrix proteins, and it participates in the activation of different pro-MMP.

Hydrogen sulfide $\left(\mathrm{H}_{2} \mathrm{~S}\right)$ is a small gaseous molecule that has been recognized as an endogenous gasotransmitter [5,6]. Its discovery in the late 90 's as an endogenous signaling molecule in the nervous and cardiovascular systems, triggered the research on its mechanisms of action and its effects in different pathologies, which hasn't stopped growing since [7-11].

The study of the functions of $\mathrm{H}_{2} \mathrm{~S}$ in the musculoskeletal system is quite incipient. Yet, it is already accepted that it is present and modulated in the osteoarthritic process [12-14] and it exerts anti-inflammatory and cytoprotective effects in joint cells at low micromolar doses $[12,14,15]$. In 2010, Whiteman et al. showed that $\mathrm{H}_{2} \mathrm{~S}$ is present in the synovial fluid (SF). In patients with rheumatoid arthritis, $\mathrm{SF} \mathrm{H}_{2} \mathrm{~S}$ levels were significantly and substantially higher than in paired plasma samples or $\mathrm{SF}$ aspirates from OA patients [14]. Additionally, $\mathrm{H}_{2} \mathrm{~S}$ levels in $\mathrm{SF}$ and plasma correlated with clinical markers of disease activity and inflammation. Later, the expression of $\mathrm{H}_{2} \mathrm{~S}$ synthesis enzymes cystathionine- $\beta$-synthase (CBS) and cystathionine- $\gamma$-lyase (CTH) was demonstrated in chondrocytes and chondrogenically-differentiated mesenchymal stem cells [12]. Lastly, several groups including ours have found that exogenous administration of $\mathrm{H}_{2} \mathrm{~S}$ to OA chondrocytes exerts anti-inflammatory and anti-catabolic effects after $24-48 \mathrm{~h}$ of stimulation [15-17]. Reduced gene 
expression and protein abundance of inflammation mediator such as IL-6, MMP3 and 13, NO or prostaglandin $\mathrm{E}_{2}$ was demonstrated.

These studies indicate that not only is $\mathrm{H}_{2} \mathrm{~S}$ present endogenously in the joint, but that in OA cartilage exogenous administration can be beneficial. However, only short term effects were assessed while cartilage degradation is a long-term process.

Therefore, the aim of the present work was to evaluate the ability of two compounds that produce $\mathrm{H}_{2} \mathrm{~S}$, sodium hydrosulfide $(\mathrm{NaSH})$ and GYY4137 (morpholin-4-ium 4 methoxyphenyl(morpholino phosphinodithioate)), to counteract the effects of IL-1 $\beta$ on matrix destruction. In particular, experiments were performed to qualitatively and quantitatively assess effects on the principal matrix component through tissue histology and immunohistochemistry, and to quantify GAGs destruction in cartilage stimulated with IL-1 $\beta$ for 21 days.

\section{Materials and methods}

\subsection{Materials}

Dulbecco's Modified Eagle Medium (DMEM), fetal bovine serum (FBS) and Penicillin/streptomycin (P/S, 100units/ml and $100 \mu \mathrm{g} / \mathrm{ml}$, respectively) were from Gibco (Gibco, Madrid, Spain). Culture flasks, well plates and other disposable culture plastic were purchased from BD (BD Bioscience, Madrid, Spain). Sodium hydrosulfide (NaSH) and human recombinant IL-1 $\beta$ were purchased from Sigma-Aldrich (Sigma-Aldrich Química S.A, Madrid Spain). GYY4137 (morpholin-4-ium 4 methoxyphenyl(morpholino phosphinodithioate)) was purchased from Santa Cruz Biotechnology, Heidelberg, Germany. Antibodies and conditions used for IHC are in Table 1. The GAGs quantification assay (Blyscan) was from Scharlab S.L. (Barcelona, Spain).

Table 1. List and characteristics of the antibodies used in the immunohistochemistry techniques. All were mouse anti-human monoclonal antibodies.

\begin{tabular}{|c|c|c|c|c|}
\hline Antibodies & Clon Isotype & Provider & Pretreatment & Dilution \\
\hline Collagen type II & $5 \mathrm{~B} 2.5 \mathrm{IgG}_{1} \kappa$ & Abcam $^{\circledR}$ & Chondroitinase $\mathrm{ABC}$ pH 8.0 & $1: 25$ \\
\hline Chondroitin 6-sulfate & CS-56 IgM & Abcam $^{\circledR}$ & Chondroitinase $\mathrm{ABC}$ pH 6.8 & $1: 100$ \\
\hline Aggrecan & BC-3 IgG & Abcam $^{\circledR}$ & Chondroitinase $\mathrm{ABC}$ pH 6.8 & $1: 100$ \\
\hline Keratan sulfate & 4B3/D10 $\mathrm{IgG}_{1}$ & Santa Cruz & none & $1: 100$ \\
\hline Matrix metalloproteinase 3 & SL-1 IID4 IgG2b & Millipore & Chondroitinase $\mathrm{ABC}$ pH 6.8 & $1: 25$ \\
\hline Matrix metalloproteinase 13 & VIIIA $2 \operatorname{IgG}_{1} \kappa$ & Thermo Scientific & Proteinase $\mathrm{K}$ & $1: 50$ \\
\hline
\end{tabular}

Reagents for tissue histology were from either Merck or Sigma-Aldrich, except for alcian blue (AB) (Applichem, VWR International Eurolab S.L. Barcelona, Spain) and DePex (Gurr ${ }^{\circledR}$, VWR International Eurolab S.L. Barcelona, Spain). Those for immunohistochemistry (IHC) were from Dako (Dako, Barcelona, Spain). 


\subsection{Tissue selection and isolation}

Osteoarthritic cartilage was obtained from samples included in the Sample Collection for Research on Rheumatic Disease started by Dr. Francisco Blanco García. This collection was authorised by the Galician Research Ethics Committee (Comité Autonómico de Ética da Investigación de Galicia, CAEIG) with registry code 2013/107 and has been inscribed in the National Registry of Biobanks (Registro Nacional de Biobancos), Collections Section code: C.0000424. Informed consent was obtained from all the donors.

Cartilage used in this study was obtained from 1 female and 2 male donors aged 74, 78 and 64, respectively, at the time of the surgery. All had been diagnosed with osteoarthritis.

\subsection{Exposure of cartilage to inflammatory mediators}

Cartilage tissue was isolated from femoral heads or condyles, or from tibial plateaus by resection with a scalpel. The sheets of cartilage were then cut into discs with a $6 \mathrm{~mm}$ biopsy punch, so that cartilage discs dimensions were approximately $6 \mathrm{~mm}$ in diameter and $2 \mathrm{~mm}$ in height. Disks were then incubated in a 24 well plate with DMEM containing $5 \%$ glutamine $(2 \mathrm{mM}), 5 \% \mathrm{P} / \mathrm{S}$, and $10 \%$ FBS in a humidified incubator with $5 \% \mathrm{CO}_{2}$ at $37{ }^{\circ} \mathrm{C}$ for $24 \mathrm{~h}$.

Experiments were performed to investigate if co-estimulation with $\mathrm{H}_{2} \mathrm{~S}$ could protect matrix components and reduce matrix destruction in cartilage stimulated with IL-1 $\beta$. For this end, cartilage explants (two per condition) were stimulated with $5 \mathrm{ng} / \mathrm{ml}$ of IL-1 $\beta$ and NaSH or GYY4137 (200 or $1000 \mu \mathrm{M}$ ), keeping one unstimulated condition (basal) and one condition with only $5 \mathrm{ng} / \mathrm{mL}$ of IL- $1 \beta$. The medium (containing the stimuli) was renewed every 4 days for a total of 21 days. Supernatants were collected with each medium change and stored at $-80{ }^{\circ} \mathrm{C}$ for further analyses.

After the stimulation period, tissue discs were recovered, carefully patted dry with a piece of filter paper and weighed. One half of each disc was processed for histology and immunohistochemistry, and the other two halves were used for GAGs quantification assays.

\subsection{Glycosaminoglycans release and quantification}

For each condition, total glycosaminoglycans content was quantified by measuring the (accumulative) amount released to the supernatants and the remaining amount in the explants. A commercial kit, Blyscan sulfated glycosaminoglycans assay, was used for both, tissue and medium GAGs quantification, following the protocol described therein. Briefly, for quantification in the supernatants, $1 \mathrm{ml}$ of Blyscan dye reagent was added to $100 \mu \mathrm{l}$ of each sample supernatant (final volume including dilutions, if necessary). This was mixed by inversion and tubes were gently shaken for $30 \mathrm{~min}$. This causes a sulfated GAG-dye complex to form and precipitate from the solution. Tubes were centrifuged $12000 \mathrm{rpm}$ for $10 \mathrm{~min}$ and the supernatant discarded. After this, $0.5 \mathrm{ml}$ of dissociation reagent was added to the tubes, vortex mixed and centrifuged again at $12000 \mathrm{rpm}$ for $5 \mathrm{~min}$, to remove foam. Then, $200 \mu \mathrm{l}$ of each resulting supernatant were transferred to a 96 -well plate and read at $656 \mathrm{~nm}$ in a Nanoquant Infinite M200 spectrophotometer (Tecan). To analyze its GAG content the tissue was dehydrated and digested with papain. About 13-15 mg of cartilage tissue per sample was digested in $1 \mathrm{ml}$ of papain extraction reagent in a thermoblock at $65{ }^{\circ} \mathrm{C}$ overnight. When the cartilage was totally digested the solution was centrifuged at $10000 \mathrm{~g}$ for $10 \mathrm{~min}$ to collect the supernatant, the general protocol of the assay was completed and the absorbance also read. Samples were compared to a calibration curve prepared with a GAGs standard (bovine tracheal chondroitin 4 sulfate) provided by the kit. 


\subsection{Cartilage histology and immunohistochemistry analyses}

For the different studies one half of one disc of cartilage was fixed in $4 \%$ paraformaldehyde for $24 \mathrm{~h}$, dehydrated in graded alcohols $\left(70^{\circ}, 96^{\circ}\right.$ and absolute) and included in paraffin. Paraffin blocks were cut in $4 \mu \mathrm{m}$ sections, deparaffinized $\left(10 \mathrm{~min}\right.$ in an oven at $37^{\circ} \mathrm{C}$ ), rinsed with xylene for $10 \mathrm{~min}$ and rehydrated in a sequence of decreasing alcohols followed by distilled water. Sections were used for tissue histology and for immunohistochemistry.

Cartilage matrix main components were demonstrated with the following stains:

Safranin O (S-O) stain binds to sulfated GAGs such as chondroitin sulfate (ChS) and keratan sulfate (KS), so a strong red staining indicates a high amount of sulfated GAGs. Toluidine blue (TB) is a basic thiazine metachromatic dye with high affinity for acidic tissue components, and in cartilage a purple color indicates a high amount of hyaluronic acid (HA), its most abundant metachromatic component. Alcian blue is used to stain acidic glycosaminoglycans, such as chondroitin sulfate (ChS) and HA, and the peryodic acid-Schiff (PAS) staining is mainly used for staining structures containing a high proportion of carbohydrate macromolecules. In Masson's trichrome (MT) three dyes are employed to selectively stain muscle, collagen fibers, fibrin, and erythrocytes. Nuclei appear black, cytoplasm, muscle, erythrocytes are stained red and collagen is stained in blue.

In the sections for IHC the appropriate antigen-epitope retrieval pretreatment was performed for each antibody (Table 1). Endogenous peroxidase activity was blocked with Dako Real ${ }^{\mathrm{TM}}$ peroxidase blocking solution for $10 \mathrm{~min}$ at room temperature. Cells were then washed with phosphate buffer solution (PBS) and incubated with the primary antibody (Table 1). The rabbit/mouse peroxidase/DAB DAKO REAL ${ }^{\mathrm{TM}}$ EnVision $^{\mathrm{TM}}$ detection kit was used to determine antigen-antibody interactions. Slides were dehydrated in graded alcohol, cleared in xylene and mounted in DePeX. A negative control was included by omitting the primary antibody in one of the sections. All slides were then visualized in an Olympus Dx61 optical microscope, and quantified using analySIS D software (Olympus España S.A.U., Barcelona, Spain). Proteins selected for the analysis of matrix components were col II, ChS, agg, and KS, and those chosen for the analysis of catabolism were MMP3 and 13.

\section{Statistical analysis}

All experiments were performed with tissue from at least two different donors $(n \geq 2)$ and with technical duplicates in the case of the GAGs quantification assay. Quantification values in the histology and IHC stains resulted from 3 representative images (20× magnification) obtained in tissue from 2 donors, i.e mean of 6 images per condition. The results are expressed as mean \pm standard error (SE). All the statistical analyses were performed using the R software (version 2.15.2) [18]. Data were assayed with analysis of variance (ANOVA) followed via a Tukey test for multiple comparisons, except when they failed tests of normality (Shapiro-Wilk's) or homoscedasticity (Bartlett test or Levene's). In this case a Kruskal-Wallis rank sum test was performed followed by multiple comparisons. In all cases, differences were considered significant when $p<0.05$. 


\section{Results}

\subsection{Glycosaminoglycan quantification}

The results of the biochemical quantification of GAGs retained in, and released from cartilage stimulated with IL-1 $\beta$ are presented in Fig. 1. In basal conditions after 21 days, $79.9 \pm 9.5 \%$ of GAGs remained in the OA tissue, while $20.1 \pm 9.5 \%$ was released to the supernatant. As expected, exposure to IL-1 $\beta$ for 21 days resulted in a significant decrease in the amount of GAGs in the tissue $(45.5 \pm 1.9 \%)$ and a corresponding increase in the amount lost to the supernatant $(54.5 \pm 1.9 \%)$, $p<0.05$. Interestingly, co-stimulation with the $\mathrm{H}_{2} \mathrm{~S}$ forming compounds resulted in a marked improvement in all cases, increasing the amount of GAGs retained in the tissue and protecting or inhibiting their loss to the supernatant. The best condition was $200 \mu \mathrm{M} \mathrm{NaSH}(70.9 \pm 5.1$ and $29.1 \pm 5.1$, respectively) and none of them were significantly different from either the Basal or the condition with IL-1 $\beta$ only.

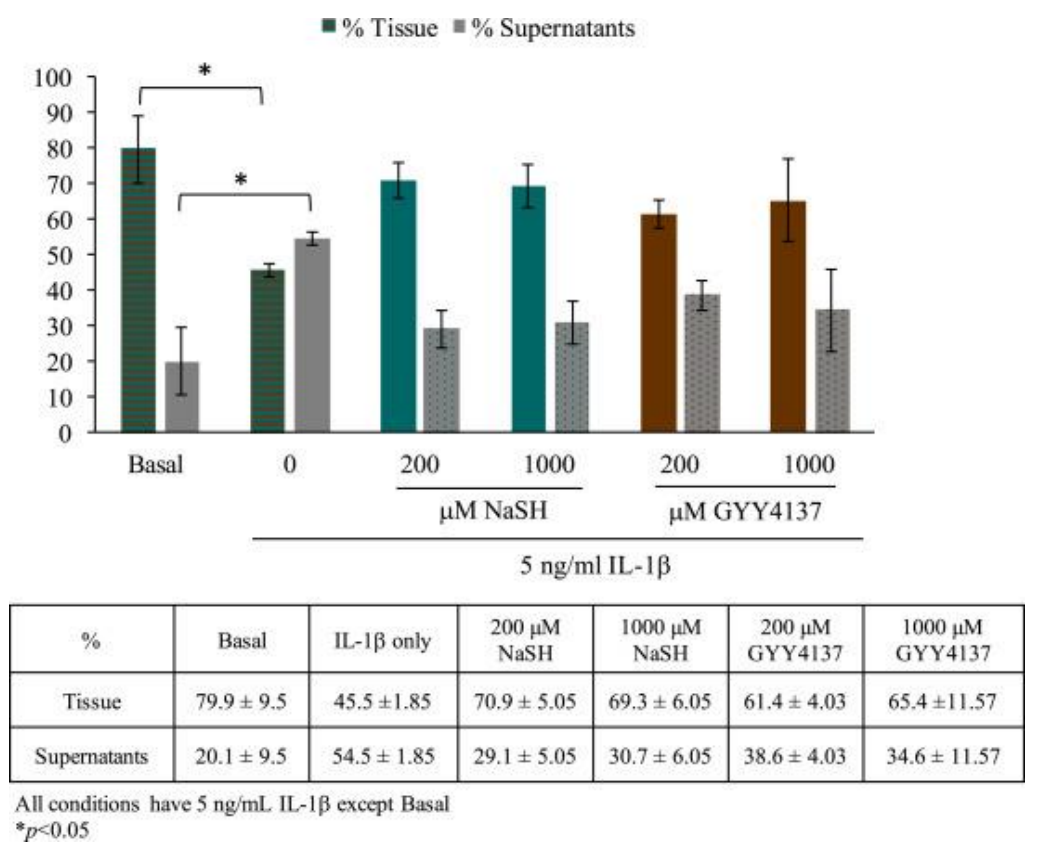

Fig. 1. Glycosaminoglycans (GAGs) percentages retained and released into the supernatant in OA cartilage after 21 days of costimulation with IL-1 $\beta$ and NaSH or GYY4137 in vitro. The percentages remaining in the tissue and released into the supernatant are shown in dark and clear bars, respectively.

\subsection{Histological stainings}

Representative images of the different histological stains performed are presented in Fig. 2. Quantification of the images was performed as explained in the methods. Results are included in Table 2, and summarized below. 

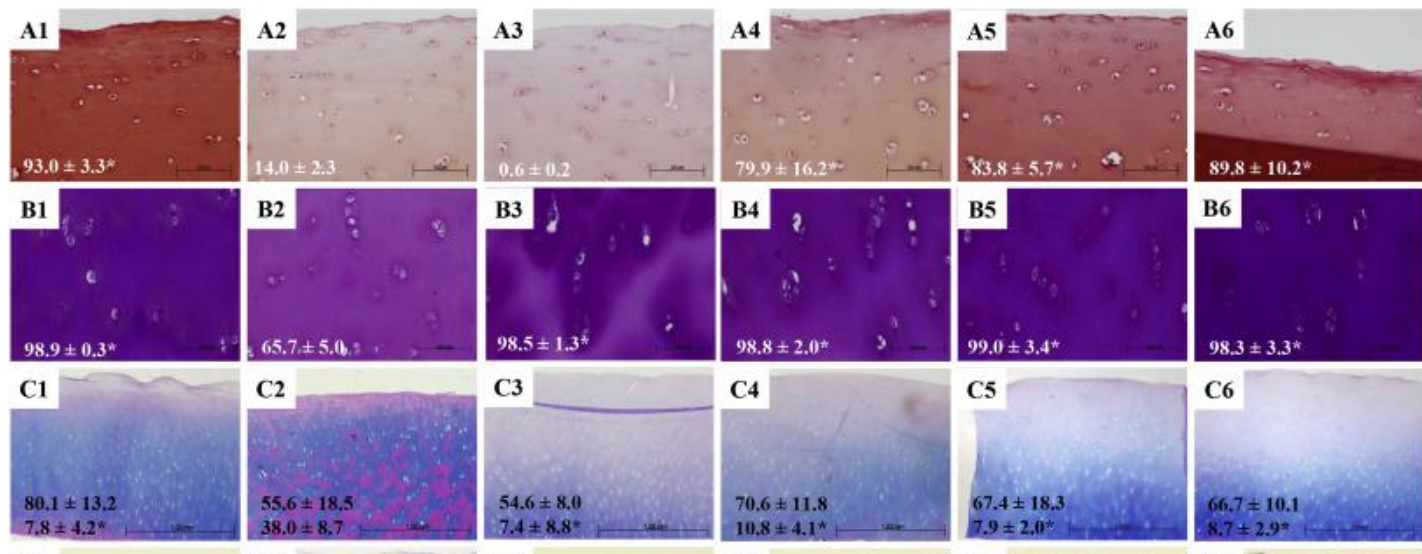

C3

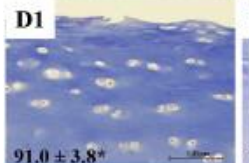

D2

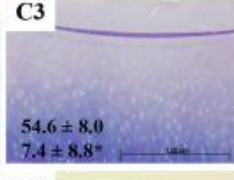

C4

B5

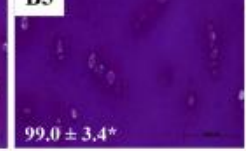

B6
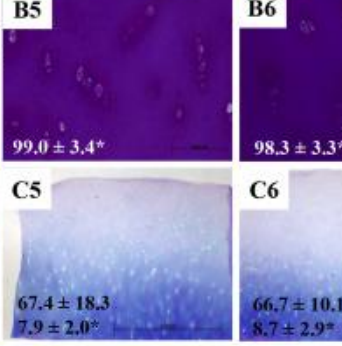

C6

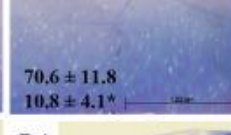

D5

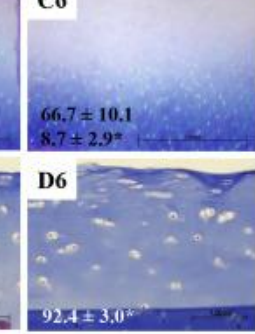

\begin{tabular}{|c||c|c|c|c|c|}
\hline \multirow{2}{*}{ BASAL } & $0 \mu \mathrm{M}$ & $200 \mu \mathrm{M} \mathrm{NaSH}$ & $1000 \mu \mathrm{M} \mathrm{NaSH}$ & $200 \mu \mathrm{M}$ GYY4 4137 & $1000 \mu \mathrm{M}$ GYY4137 \\
\cline { 2 - 4 } & $5 \mathrm{ng} / \mathrm{mL} \mathrm{IL}-1 \beta$ \\
\hline
\end{tabular}

Fig. 2. Representative images of histological stains performed in OA cartilage after 21 days of co-stimulation with IL- $1 \beta$ and $\mathrm{NaSH}$ or GYY4137 in vitro. A) Safranin O, B) Toluidine blue, C) Alcian blue - PAS, D) Masson's trichrome. Numbers represent the following conditions: 1) Basal, 2) Stimulation with IL-1 $\beta$ only, 3) IL- $1 \beta+200 \mu \mathrm{M} \mathrm{NaSH}, 4) \mathrm{IL}-1 \beta+1000 \mu \mathrm{M}$ $\mathrm{NaSH}$, 5) IL-1 $\beta+200 \mu \mathrm{M}$ GYY4137, 6) IL-1 $\beta+1000 \mu \mathrm{M}$ GYY4137. In A), B) and D) size bars represent $100 \mu \mathrm{m}$, in C, 1 mm. $* p<0.05$ with respect to the condition with IL-1 $\beta$.

Table 2. Quantifications for safranin O, toluidine blue, alcian blue-PAS and Masson's trichrome staining performed in osteoarthritic cartilage stimulated with IL- $1 \beta .{ }^{*} p<0.05$ with respect to the condition with IL- $1 \beta$ only.

\begin{tabular}{|c|c|c|c|c|c|c|}
\hline \multirow{3}{*}{ Stain } & \multirow{3}{*}{ Basal } & \multicolumn{5}{|c|}{ All conditions stimulated with IL- $1 \beta(5 \mathrm{ng} / \mathrm{mL})$} \\
\hline & & \multirow[t]{2}{*}{$0 \mu \mathrm{M}$} & \multicolumn{2}{|l|}{$\mathrm{NaSH}$} & \multicolumn{2}{|l|}{ GYY4137 } \\
\hline & & & $200 \mu \mathrm{M}$ & $1000 \mu \mathrm{M}$ & $200 \mu \mathrm{M}$ & $1000 \mu \mathrm{M}$ \\
\hline Safranin $\mathrm{O}$ & $93.0 \pm 3.3^{*}$ & $14.0 \pm 2.3$ & $0.6 \pm 0.2$ & $79.9 \pm 16.2 *$ & $83.8 \pm 5.7 *$ & $89.8 \pm 10.2 *$ \\
\hline Toluidine blue & $98.9 \pm 0.3^{*}$ & $65.7 \pm 5.0$ & $98.5 \pm 1.3^{*}$ & $98.8 \pm 2.0^{*}$ & $99.0 \pm 3.4^{*}$ & $98.3 \pm 3.3^{*}$ \\
\hline Alcian blue PAS & $\begin{array}{l}80.1 \pm 13.2 \\
7.8 \pm 4.2^{*}\end{array}$ & $\begin{array}{l}55.6 \pm 18.5 \\
38.0 \pm 8.7\end{array}$ & $\begin{array}{l}54.6 \pm 8.0 \\
7.4 \pm 8.8^{*}\end{array}$ & $\begin{array}{l}70.6 \pm 11.8 \\
10.8 \pm 4.1^{*}\end{array}$ & $\begin{array}{l}67.4 \pm 18.3 \\
7.9 \pm 2.0^{*}\end{array}$ & $\begin{array}{l}66.7 \pm 10.1 \\
8.7 \pm 2.9^{*}\end{array}$ \\
\hline Masson's trichrome & $91.0 \pm 3.8^{*}$ & $79.8 \pm 7.4$ & $90.1 \pm 2.1 *$ & $90.0 \pm 0.2 *$ & $90.5 \pm 5.8 *$ & $92.4 \pm 3.0^{*}$ \\
\hline
\end{tabular}

\subsubsection{Safranin $O$}

S-O staining (Fig. 2A and Table 2) revealed a sharp loss of GAGs due to the cytokine exposure from $93.0 \pm 3.3$ in the basal condition down to $14.0 \pm 2.3$ in the IL-1 $\beta$ only condition (Fig. $2 \mathrm{~A} 1$ and $2 \mathrm{~A} 2$, respectively). It is also striking that in the basal condition (Fig. 2A1), the inter-territorial region surrounding chondrocytes was more positive than the rest of the matrix, suggesting that cells were actively producing GAGs, while in the discs stimulated with IL-1 $\beta$ this positivity was lost. GAGs were protected when the tissue was co-stimulated with 200 and $1000 \mu \mathrm{M}$ GYY4137 doses $(83.8 \pm 5.7 \%$ and $89.8 \pm 10.2 \%$, respectively Fig. 2A5-6), whereas in the case of NaSH only the highest concentration exerted this effect $(98.5 \pm 1.3 \%$, Fig. 2A3-4). Quantitatively, all conditions were significantly better than IL-1 $\beta$ ( $p<0.05$ ), except for $200 \mu \mathrm{M} \mathrm{NaSH}$ (Table 2). 


\subsubsection{Toluidine blue}

In the staining for metachromatic components, represented in cartilage mostly by HA, no differences in staining intensity were observed in the superficial layer (not shown), and the effects of both IL-1 $\beta$ and the sulfide compounds were more apparent in the deeper layer of cartilage, so we opted for showing these areas instead. Staining was reduced from $98.9 \pm 0.3$ to $65.7 \pm 5.0$ when OA tissue was cultured with IL-1 $\beta$ (Fig. 2B1 vs. 2B2, Table 2). This effect was counteracted by both $\mathrm{H}_{2} \mathrm{~S}$-forming compounds and a concentration of $200 \mu \mathrm{M}$ of either seemed already fully effective, recovering the basal levels (Fig. 2B3-6, Table $2 ; p<0.05$ vs. IL-1 $\beta$ ). Further, the inter-territorial and intra-cellular regions that had practically lost their staining with IL-1 $\beta$, also recovered with $\mathrm{NaSH}$ and GYY4137 co-estimulation.

\subsubsection{Alcian blue-PAS}

It is noteworthy that there were small areas of intense PAS staining (fuchsia) in the deep zone of cartilage (visible in the lower left corners of Fig. $2 \mathrm{C} 1$ and 2C6) that were remarkably increased due to IL-1 $\beta$ stimulation $(7.8 \pm 4.2 \%$ in basal condition and $38.0 \pm 8.7 \%$ in IL-1 $\beta$ condition [Fig. 2C2] and Table 2). This was not seen in normal cartilage (results not shown). This suggests that there is an increased presence of carbohydrates in OA, and that its production is induced by IL-1 $\beta$. On the other hand, we also observed a slight loss of alcian blue staining in all OA tissue exposed to IL-1 $\beta$, from $80.1 \pm 13.2$ (basal) down to $55.6 \pm 18.5$ (IL-1 $\beta$ only).

Co-stimulation with NaSH or GYY4137 reduced PAS staining almost to baseline ( $<10 \%)$, evidencing no clear differences in different doses nor in using NaSH or GYY4137 (Fig. 2C3-6 and Table 2). As for the AB staining, there seems to be a recovery with all the conditions tested.

\subsubsection{Masson's trichrome}

OA cartilage stimulated with IL-1 $\beta$ was only slightly less positive than the unstimulated condition $(79.8 \pm 7.4 \%$, Fig. 2D2 and $91.0 \pm 3.8 \%$, Fig. 2D1, respectively), and adding different concentrations of $\mathrm{NaSH}$ or GYY4137 completely prevented this loss.

\subsection{Immunohistochemical analyses}

\subsubsection{Matrix proteins}

Cartilage most abundant matrix proteins, col II and agg plus the main sulfated GAGs, KS and $\mathrm{ChS}$, were also revealed and quantified with immunohistochemistry. Representative images of the different determinations are presented in Fig. 3. Quantification of the images was done as explained in the methods. Results are included in Table 3. 


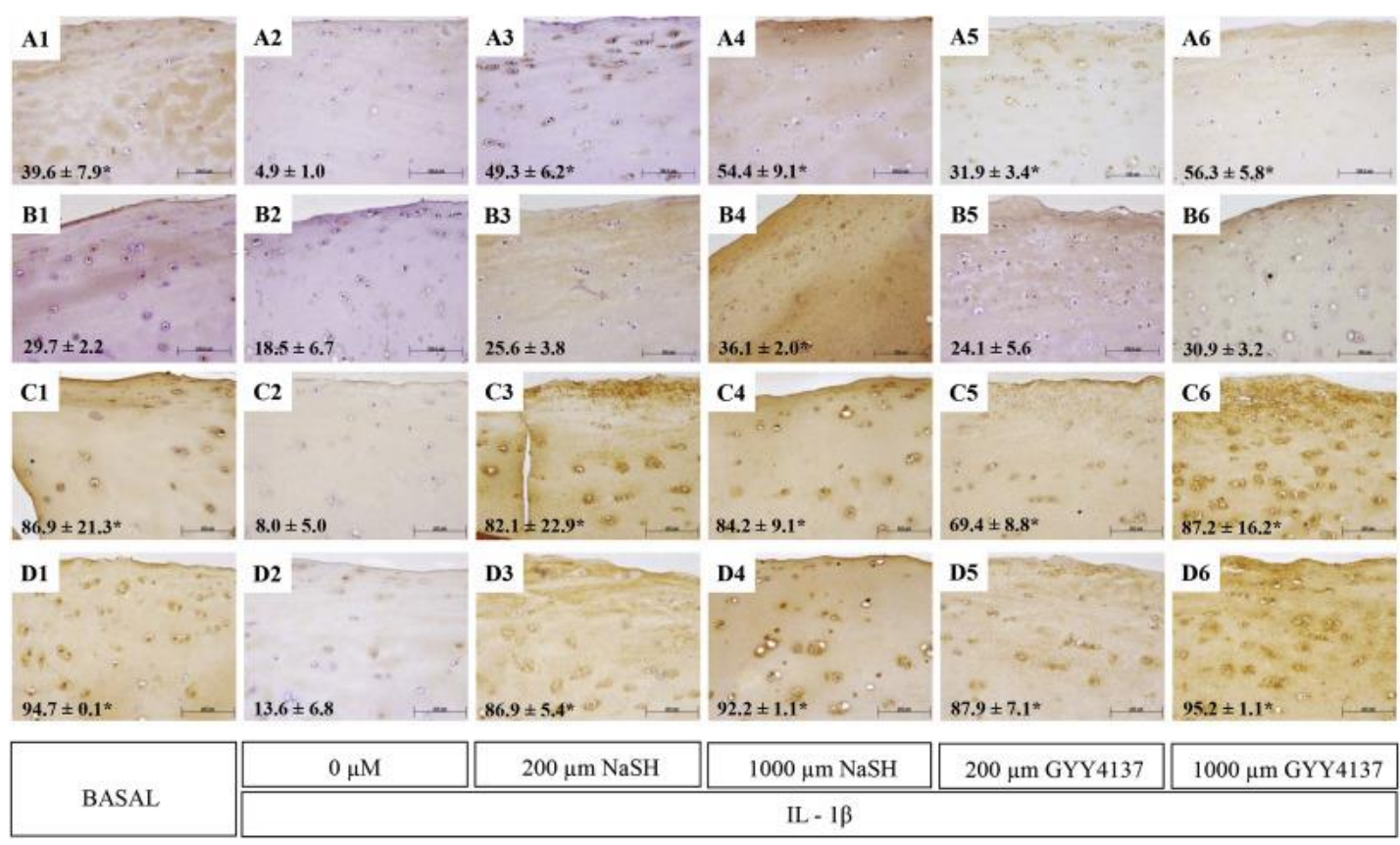

Fig. 3. Representative images of immunohistochemical stains performed in OA cartilage after 21 days of co-stimulation with IL-1 $\beta$ and NaSH or GYY4137 in vitro. A) Collagen type II, B) Aggrecan, C) Keratan sulfate, D) Chondroitin sulfate. Numbers represent the following conditions: 1) Basal, 2) Stimulation with IL-1 $\beta$ only, 3) IL-1 $\beta+200 \mu \mathrm{M}$ NaSH, 4) IL-1 $\beta+1000 \mu \mathrm{M}$ NaSH, 5) IL-1 $\beta+200 \mu \mathrm{M}$ GYY4137, 6) IL-1 $\beta+1000 \mu \mathrm{M}$ GYY4137. All size bars represent $100 \mu \mathrm{m}$. * $p<0.05$ with respect to the condition with IL-1 $\beta$.

Table 3. Quantifications for the immunohistochemical determinations of collagen type II, aggrecan, keratan sulfate, and chondroitin sulfate performed in osteoarthritic stimulated with IL-1 $\beta .{ }^{*} p<0.05$ with respect to the condition with IL- $1 \beta$ only.

\begin{tabular}{|c|c|c|c|c|c|c|}
\hline \multirow{3}{*}{ Protein } & \multirow{3}{*}{ Basal } & \multicolumn{5}{|c|}{ All conditions stimulated with IL- $1 \beta(5 \mathrm{ng} / \mathrm{mL})$} \\
\hline & & \multirow[t]{2}{*}{$0 \mu \mathrm{M}$} & \multicolumn{2}{|l|}{$\mathrm{NaSH}$} & \multicolumn{2}{|l|}{ GYY4137 } \\
\hline & & & $200 \mu \mathrm{M}$ & $1000 \mu \mathrm{M}$ & $200 \mu \mathrm{M}$ & $1000 \mu \mathrm{M}$ \\
\hline Collagen type II & $39.6 \pm 7.9^{*}$ & $4.9 \pm 1.0$ & $49.3 \pm 6.2^{*}$ & $54.4 \pm 9.1 *$ & $31.9 \pm 3.4 *$ & $56.3 \pm 5.8^{*}$ \\
\hline Aggrecan & $29.7 \pm 2.2$ & $18.5 \pm 6.7$ & $25.6 \pm 3.8$ & $36.1 \pm 2.0^{*}$ & $24.1 \pm 5.6$ & $30.9 \pm 3.2$ \\
\hline Keratan sulfate & $86.9 \pm 21.3^{*}$ & $8.0 \pm 5.0$ & $82.1 \pm 22.9^{*}$ & $84.2 \pm 9.1^{*}$ & $69.4 \pm 8.8^{*}$ & $87.2 \pm 16.2 *$ \\
\hline Chondroitin sulfate & $94.7 \pm 0.1 *$ & $13.6 \pm 6.8$ & $86.9 \pm 5.4^{*}$ & $92.2 \pm 1.1^{*}$ & $87.9 \pm 7.1^{*}$ & $95.2 \pm 1.1 *$ \\
\hline
\end{tabular}

The IHC analyses of col II (Fig. 3A) and agg (Fig. 3B) in the unstimulated tissue showed $39.6 \pm 7.9 \%$ and $29.7 \pm 2.2 \%$ positivity, respectively and that IL-1 $\beta$ was responsible for significant losses of both cartilage ECM components, resulting in positivities of $4.9 \pm 1.0 \%$ and $18.5 \pm 6.7 \%$ respectively (Fig. 3A2 and B2, Table 3). However, when tissue was co-stimulated with $\mathrm{NaSH}$ or GYY4137 (Fig. 3A3-6 and 3B3-6), not only was their loss prevented, but levels were in fact higher than in the basal condition (Table 3). 
On the other hand, the analyses for keratan and chondroitin sulfates (Fig. 3C and D, respectively; and Table 3) indicated that the loss in GAGs seen with the other methods (quantification kit and S-O and $A B$ stains) in the IL- $1 \beta$ conditions is, at least in part, due to a loss in these sulfated GAGs. Keratan sulfate positivity was reduced from $86.9 \pm 21.5 .0$ to $8.0 \pm 5.0$ and chondroitin sulfate from $94.7 \pm 0.1$ to $13.6 \pm 6.8$ (Basal vs IL-1 $\beta$ only, respectively). In both cases, the co-stimulation of IL-1 $\beta$ with NaSH or GYY4137 led to significant improvements, recovering positivity percentages similar to those observed in the basal condition (Fig. 3C3-6 and D3-6 and Table 3). It also stands out, again focusing in the inter-territorial region, that $\mathrm{KS}$ (and $\mathrm{ChS}$ to a lesser extent) production was lost in this area due to IL-1 $\beta$, but it was recovered with the co-stimulation with NaSH or GYY4137.

\subsubsection{Metalloproteinases}

In addition, we also performed MMP3 and 13 IHCs in the cartilage disks (Fig. 4A and B, respectively, and Table 4). With respect to MMP3, the protein was already present in the basal condition $(4.0 \pm 1.0 \%)$, although the positivity was low (Fig. 4A1) and it increased markedly after exposure to IL-1 $\beta(84.2 \pm 8.2 \%$, Fig. 4A2). Co-stimulation with $\mathrm{NaSH}$ or GYY4137 caused an attenuation of this effect in all cases but only $1000 \mu \mathrm{M} \mathrm{NaSH}(41.4 \pm 7.3 \%), 200 \mu \mathrm{M}$ GYY4137 $(33.2 \pm 2.9 \%)$ and $1000 \mu \mathrm{M}$ GYY4137 $(12.5 \pm 3.5 \%)$ were significantly lower $(p<0.05)$. On the other hand, positivity inside the cells was only abated with the highest concentration (Fig. 4A4 and $6)$. In the case of MMP13, its presence in the basal condition was very low $(40.5 \pm 0.2 \%$ Fig. $4 \mathrm{~B} 1$, Table 4) but, chondrocytes actively synthesized this collagenase as a result of IL-1 $\beta$ stimulation $(10.4 \pm 3.2 \%$, Fig. 4B2) and GYY4137 was more effective in counteracting this effect $(200 \mu \mathrm{M}$ : $0.3 \pm 0.1 \%$ and $1000 \mu \mathrm{M}: 1.0 \pm 0.5 \%)$ than $\mathrm{NaSH}(200 \mu \mathrm{M}: 7.6 \pm 3.6 \%$ and $1000 \mu \mathrm{M}: 4.3 \pm 0.1 \%)$, Fig. 4B3-6, Table 4.

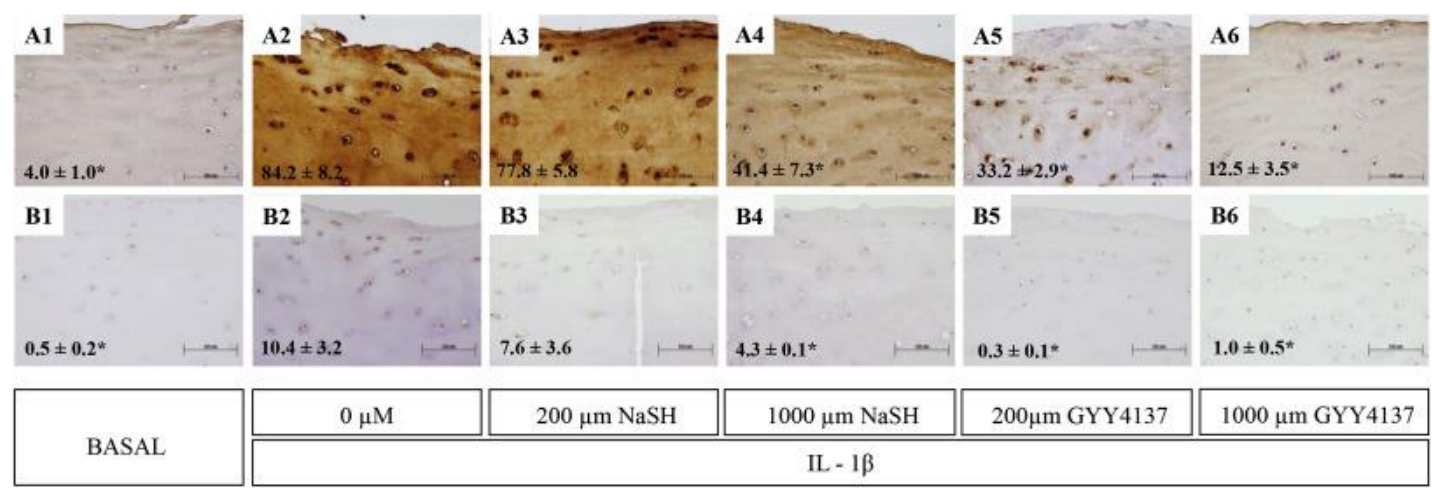

Fig. 4. Representative images of immunohistochemical stains performed in OA cartilage after 21 days of co-stimulation with IL-1 $\beta$ and NaSH or GYY4137 in vitro. A) Matrix metalloproteinase 3, B) Matrix metalloproteinase 13. Numbers represent the following conditions: 1) Basal, 2) Stimulation with IL-1 $\beta$ only, 3) IL-1 $\beta+200 \mu \mathrm{M} \mathrm{NaSH}, 4) \mathrm{IL}-1 \beta+1000 \mu \mathrm{M}$ NaSH, 5) IL$1 \beta+200 \mu \mathrm{M}$ GYY4137, 6) IL- $1 \beta+1000 \mu \mathrm{M}$ GYY4137. All size bars represent $100 \mu \mathrm{m}$. $* p<0.05$ with respect to the condition with IL-1 $\beta$. 
Table 4. Quantifications for the immunohistochemical determinations of matrix metalloproteinases (MMP) 3 and 13 performed in osteoarthritic cartilage stimulated with IL-1 $\beta$. ${ }^{*} p<0.05$ with respect to the condition with IL-1 $\beta$ only.

\begin{tabular}{|c|c|c|c|c|c|c|}
\hline \multirow{3}{*}{ Protein } & \multirow{3}{*}{ Basal } & \multicolumn{5}{|c|}{ All conditions stimulated with IL-1 $\beta$ ( $5 \mathrm{ng} / \mathrm{mL})$} \\
\hline & & \multirow[t]{2}{*}{$0 \mu \mathrm{M}$} & \multicolumn{2}{|l|}{$\mathrm{NaSH}$} & \multicolumn{2}{|l|}{ GYY4137 } \\
\hline & & & $200 \mu \mathrm{M}$ & $1000 \mu \mathrm{M}$ & $200 \mu \mathrm{M}$ & $1000 \mu \mathrm{M}$ \\
\hline MMP3 & $4.0 \pm 1.0^{*}$ & $84.2 \pm 8.2$ & $77.8 \pm 5.8$ & $41.4 \pm 7.3 *$ & $33.2 \pm 2.9 *$ & $12.5 \pm 3.5^{*}$ \\
\hline MMP13 & $0.5 \pm 0.2^{*}$ & $10.4 \pm 3.2$ & $7.6 \pm 3.6$ & $4.3 \pm 0.1^{*}$ & $0.3 \pm 0.1^{*}$ & $1.0 \pm 0.5^{*}$ \\
\hline
\end{tabular}

\section{Discussion}

The present work evaluated the effects of $\mathrm{H}_{2} \mathrm{~S}$-releasing compounds, NaSH and GYY4137 on the anabolic-catabolic balance of MEC components in OA cartilage stimulated with IL-1 $\beta$. We looked at the release of GAGs from tissue disks with a GAG quantification kit and other matrix proteins through histology and immunohistochemistry. This is the first study that we know of that investigated the involvement of $\mathrm{H}_{2} \mathrm{~S}$ in these aspects of $\mathrm{OA}$ progression directly on human cartilage tissue.

Proper maintenance of hyaline articular cartilage relies on a balance between the anabolic and catabolic processes in chondrocytes. With the onset of OA, this balance is lost and cartilage degradation supersedes its synthesis. Predictably, all assays performed in the present study demonstrated that long-term stimulation with the pro-inflammatory cytokine IL-1 $\beta$ results in loss of the main matrix components, i.e. collagen type II, aggrecan, hyaluronic acid, and sulfated GAGs. Through the GAGs quantification assay, the S-O and TB stains, and $\mathrm{KS} / \mathrm{ChS}$ IHCs it was shown that co-stimulation with $\mathrm{H}_{2} \mathrm{~S}$-forming reagents partially, or even completely in some cases, avoided GAGs destruction. We came across one exception; S-O staining for $200 \mu \mathrm{M} \mathrm{NaSH}$ suggested this concentration might be detrimental. In our opinion, this is an individual result that is refuted by the rest of the findings that support the protection of GAGs with $200 \mu \mathrm{M} \mathrm{NaSH}$.

As for the content of col II, the MT staining was not very informative. Qualitatively, the histologic results did not indicate major differences in col II content between the various conditions. However, the col II IHC allowed us to quantitatively assert a significant recovery with both sulfide compounds.

In our opinion, results also indicate that NaSH and GYY4137 might have pro-anabolic effects. In some of the histological stains and IHCs, positivity inside the cells and/or the inter-territorial zone was lost, suggesting that active synthesis by the cells was inhibited by IL-1 $\beta$. This was recovered with the $\mathrm{H}_{2} \mathrm{~S}$-forming compounds. Further, according to the IHCs, col II and agg were not only recovered but even promoted. Strictly speaking, we cannot tell if $\mathrm{H}_{2} \mathrm{~S}$ is simply protecting matrix degradation or if it is, in addition, inducing the synthesis of matrix proteins by the chondrocytes. However, quantification of the images and the mentioned effects in the intra-cellular and interterritorial regions seem to suggest the latter.

Another noteworthy finding is the increased presence of carbohydrates in the tissue, revealed in the PAS stain, that is further increased after 21 days of stimulation with IL-1 $\beta$. This could be related to a change towards a lipidic metabolism in chondrocytes which has been linked to the development of OA $[19,20]$. This increase in carbohydrates effectively disappears in the presence of NaSH or GYY4137, thus these compounds may also protect against this phenotypic change. 
With respect to the catabolic enzymes, the present results also demonstrated clear protective effects against the increased MMPs levels. IHCs for MMP3 and 13 also showed a significant increase with stimulation with IL-1 $\beta$ and were subsequently reduced with the co-stimulation with sulfide compounds, especially at the highest dose of GYY4137. The present results are in line with others previously published by our group [15]. We showed that $48 \mathrm{~h}$ co-stimulation with $\mathrm{NaSH}$ and GYY4137 had anti-catabolic effects in IL-1 $\beta$ stimulated OA chondrocytes. We observed a dosedependent reduction of gene expression of MMP3 and 13, with both compounds. This also corresponded with a reduction in protein levels, evidenced both with IHC and, in the case of MMP13, an ELISA. Here we demonstrate that this protective effect against MMPs is maintained over time and directly in cartilage tissue in vitro.

It is also known that IL-1 $\beta$ stimulates the release of ADAMTSs (a disintegrin and metalloproteinase with trombospondin motifs) 4 and 5 [21], responsible of the segmentation of proteoglycans such as aggrecan and versican [22]. In the tissue used in our study, apart from the overproduction of metalloproteinases, there surely also was an increase in these aggrecanases, reflected by the loss of cartilage GAGs from the discs and the agg IHC. However, in the conditions treated with $\mathrm{H}_{2} \mathrm{~S}$, loss of GAGs to supernatants was reduced, and aggrecan in the disks was preserved. So although we have no direct evidence, these results suggest that sulfide compounds were also capable of inhibiting the induction of aggrecanases, or of protecting against their effects, resulting in the protection of cartilage.

It has been demonstrated that chondrocytes' catabolic predisposition begins with an increase in aggrecanases followed by an increase in MMPs [23,24]. In general, there is a consensus that while aggrecan degradation in the ECM is a reversible process, induction of MMPs eventually leads to irreversible degradation of collagen type II [25], and an effective treatment needs to be able to revert the catabolic chondrocyte phenotype. Therefore, the present results suggest that exogenous administration of $\mathrm{H}_{2} \mathrm{~S}$ might be effective in accomplishing this, stopping the perpetuation of the catabolic phenotype in the cells.

There is, to date, no publication that we know of that analysed the effects of NaSH or GYY4137 on human OA cartilage degradation. Some studies can be found that look at the effects of $\mathrm{H}_{2} \mathrm{~S}$ sources in in vivo models of acute inflammation. The effects of GYY4137 [16], $\mathrm{Na}_{2} \mathrm{~S}$ [26] and Lawesson's reagent [27] were evaluated on signs and/or markers of inflammation, oxidative stress or pain in the joints of the animals, but none of these studies looked at long term effects on cartilage.

On the other hand, a report evaluated the effects of diallyl sulfide (DAS), an oil-soluble organosulphur compound extracted from garlic, in a mechanical model of OA in rabbits [28]. DAS treatment significantly reduced the total Mankin Score with respect to the vehicle treated group at week 9, as well as MMP1, MMP3, MMP13 and IL-1 $\beta$ gene expression in the DAS-treated cartilage. Notably, Benavides et al. [29] showed that garlic-derived organic polysulfides can be converted into hydrogen sulfide through a glucose-supported glutathione-dependent reaction and, importantly, that the cardiovascular protection attributed to garlic is in fact mediated by $\mathrm{H}_{2} \mathrm{~S}$.

Hydrogen sulfide has also been linked to the inhibition of fibrosis in several pathologies, such as lung, liver, kidney and heart fibrosis [30]. Zhang and co-workers attribute these effects to $\mathrm{H}_{2} \mathrm{~S}$ antioxidant, anti-apoptotic, anti-inflammatory, pro-angiogenic properties and its ability to inhibit fibroblast activation and speculate that $\mathrm{H}_{2} \mathrm{~S}$ will likely play a role in all kinds of fibrosis in the body. Cartilage might well be one example. The attempts by chondrocytes to repair damaged tissue result in the formation of fibro-cartilage, which does not have the same mechanical or compositional properties as hyaline cartilage [31].

Additionally, several reports have also provided evidence that $\mathrm{H}_{2} \mathrm{~S}$ biosynthesis or its endogenous levels are reduced or correlate with disease severity in some chronic pathologies such as hypertension [32], type II diabetes [33] or chronic obstructive pulmonary disease [34]. With respect to OA, our group has some preliminary evidence that $\mathrm{H}_{2} \mathrm{~S}$ biosynthesis might be reduced in the osteoarthritic joint [35]. We believe this might be another factor contributing to the onset of OA, though more research is needed in this direction. 
Overall, the present results indicate that hydrogen sulfide, supplied as NaSH and GYY4137, exerts tissue protective effects in OA cartilage stimulated with IL-1 $\beta$ for 21 days in vitro. $\mathrm{H}_{2} \mathrm{~S}$ appears to prevent the shift toward catabolic phenotype in chondrocytes and was able not only to protect cartilage ECM against the catabolic action potentiated by IL-1 $\beta$, but also to stimulate the synthesis of GAGs, aggrecan and type II collagen in cells.

Therefore, exogenous supplementation with sources of $\mathrm{H}_{2} \mathrm{~S}$ should be further explored as a therapeutic option for osteoarthritis. These might include synthetic reagents that produce $\mathrm{H}_{2} \mathrm{~S}$ or alternatively, natural $\mathrm{H}_{2} \mathrm{~S}$ sources, such as sulfurous mineral waters. A few recent randomized clinical trials and systematic reviews have found evidence supporting beneficial effects of balneotherapy using this kind of waters [36,37,38]. In this context, our results might also contribute to the understanding of the mechanisms responsible for these positive effects, which at present have not been fully elucidated [39]. It could be hypothesized that because $\mathrm{H}_{2} \mathrm{~S}$ bioavailability is reduced is OA, exogenous supplementation, acting through anti-inflammatory, anti-catabolic, anti-oxidant and/or anti-fibrotic pathways results in the beneficial effects that have been demonstrated in in vitro and in vivo pre-clinical models and human clinical trials.

More in vivo studies in OA animal models comparing for instance, intra-articular administration of $\mathrm{H}_{2} \mathrm{~S}$ synthetic compounds to balneotherapy in sulfurous water would provide further evidence of efficacy and help to determine the most appropriate route of administration.

\section{Acknowledgements and funding sources}

Authors want to express their gratitude to the patients and staff of the Rheumatology and Orthopedic Services of the University Hospital A Coruña (CHUAC). AVA was supported by the University de A Coruña Foundation. CVG was supported by Xunta de Galicia (Postdoctoral contract POSA/2013/206). EFB was supported by Axencia Galega de Innovación (IPP program) and Centro de Investigación Biomédica en Red-Bioingeniería, Biomateriales y Nanomedicina (CIBER-BBN).

This work was supported by CIBER-BBN and Instituto de Salud Carlos III (ISCIII, Grant PI12/00329). CIBER-BBN is a national initiative of ISCIII.

\section{Conflict of interests}

The authors declare they do not have any conflict of interest. No competing financial interests exist.

EF Burguera, S Díaz-Prado, R Meijide-Failde and FJ Blanco were involved in the conception and design of the experimental part. Á Vela-Anero, T Hermida-Gómez, C Vaamonde-Garcia and L GatoCalvo performed the data acquisition and contributed to data analysis and interpretation, together with the rest of the authors. All co-authors were involved in drafting or critically reading the manuscript and approved the final version.

\footnotetext{
Abbreviation list

AB: alcian blue; ADAMTS: a disintegrin and metalloproteinase with trombospondin motifs; Agg: aggrecan; ANOVA: analysis of variance; CBS: cystathionine-b-synthase; ChS: chondroitin sulfate; Col: collagen; CTH: cystathionine-g-lyase; DAS: diallyl sulfide; DMEM: Dulbecco's Modified Eagle Medium; ECM: extracellular matrix; FBS: fetal bovine serum; GAG: glycosaminoglycan; GYY4137: morpholin-4-ium 4 methoxyphenyl(morpholino phosphinodithioate); $\mathrm{H}_{2} \mathrm{~S}$ : hydrogen sulfide; HA: hyaluronic acid; IHC: immunohistochemistry; IL: interleukin; KS: keratan sulfate; MMP: matrix metalloproteinases; MT: Masson's trichrome; NaSH: sodium hydrosulfide; NO: nitric oxide; OA: osteoarthritis; P/S: Penicillin/streptomycin; PAS: peryodic acid-Schiff; PBS: phosphate buffer solution; runx2: Runt-related transcription factor 2; S-O: safranin-O; SE: standard error; SF: synovial fluid; TB: toluidine blue; TNF: tumor necrosis factor; WOMAC: The Western Ontario and McMaster Universities Osteoarthritis Index.
} 


\section{References}

[1] A.-C. Bay-Jensen, S. Hoegh-Madsen, E. Dam, K. Henriksen, B.C. Sondergaard, P. Pastoureau, et al. Which elements are involved in reversible and irreversible cartilage degradation in osteoarthritis?. Rheumatol. Int., 30 (2010), pp. 435-442

[2] Reimann, H.J. Mankin, C. Trahan. Quantitative histologic analyses of articular-cartilage and subchondral bone from osteoarthritic and normal human hips. Acta Orthop. Scand., 48 (1977), pp. 63-73

[3] P.S. Burrage, K.S. Mix, C.E. Brinckerhoff. Matrix metalloproteinases: role in arthritis. Front. Biosci., 11 (2006), pp. 529-543

[4] L.J. Bonassar, E.H. Frank, J.C. Murray, C.G. Paguio, V.L. Moore, M.W. Lark, et al. Changes in cartilage composition and physical-properties due to stromelysin degradation. Arthritis Rheum., 38 (1995), pp. 173-183

[5] R. Wang. Two's company, three's a crowd: can H2S be the third endogenous gaseous transmitter?. FASEB J., 16 (2002), pp. 1792-1798

[6] K. Abe, H. Kimura. The possible role of hydrogen sulfide as an endogenous neuromodulator. J. Neurosci., 16 (1996), pp. 1066-1071

[7] W.M. Zhao, J.F. Ndisang, R. Wang. Modulation of endogenous production of H2S in rat tissues. Can. J. Physiol. Pharmacol., 81 (2003), pp. 848-853

[8] W.M. Zhao, R. Wang. H2S-induced vasorelaxation and underlying cellular and molecular mechanisms. Am. J. Physiol. Heart Circ. Physiol., 283 (2002), pp. H474H480

[9] R. Wang. The gasotransmitter role of hydrogen sulfide. Antioxid. Redox Signal, 5 (2003), pp. 493-501

[10] M. Whiteman, J.S. Armstrong, S.H. Chu, S. Jia-Ling, B.S. Wong, N.S. Cheung, et al. The novel neuromodulator hydrogen sulfide: an endogenous peroxynitrite 'scavenger'? J. Neurochem., 90 (2004), pp. 765-768

[11] M. Yusuf, B.T.K. Huat, A. Hsu, M. Whiteman, M. Bhatia, P.K. Moore. Streptozotocininduced diabetes in the rat is associated with enhanced tissue hydrogen sulfide biosynthesis. Biochem. Biophys. Res. Commun., 333 (2005), pp. 1146-1152

[12] B. Fox, J.T. Schantz, R. Haigh, M.E. Wood, P.K. Moore, N. Viner, et al. Inducible hydrogen sulfide synthesis in chondrocytes and mesenchymal progenitor cells: is $\mathrm{H} 2 \mathrm{~S}$ a novel cytoprotective mediator in the inflamed joint?. J. Cell Mol. Med., 16 (2012), pp. 896-910

[13] D. Sieghart, M. Liszt, A. Wanivenhaus, H. Broell, H. Kiener, B. Kloesch, et al. Hydrogen sulphide decreases IL-1 beta-induced activation of fibroblast-like synoviocytes from patients with osteoarthritis. J. Cell Mol. Med., 19 (2015), pp. $187-$ 197

[14] M. Whiteman, R. Haigh, J.M. Tarr, K.M. Gooding, A.C. Shore, P.G. Winyard. Detection of hydrogen sulfide in plasma and knee-joint synovial fluid from rheumatoid arthritis patients: relation to clinical and laboratory measures of inflammation. Ann. N. Y. Acad. Sci., 1203 (2010), pp. 146-150

[15] E.F. Burguera, A. Vela-Anero, J. Magalhaes, R. Meijide-Failde, F. Blanco. Effect of hydrogen sulfide sources on inflammation and catabolic markers on interleukin 1 betastimulated human articular chondrocytes. Osteoarthr. Cartil., 22 (2014), pp. 1026-1035

[16] L. Li, B. Fox, J. Keeble, M. Salto-Tellez, P.G. Winyard, M.E. Wood, et al. The complex effects of the slow-releasing hydrogen sulfide donor GYY4137 in a model of acute joint inflammation and in human cartilage cells. J. Cell Mol. Med., 17 (2013), pp 365-376

[17] C. Ha, S. Tian, K. Sun, D. Wang, J. Lv, Y. Wang. Hydrogen sulfide attenuates IL-1 beta-induced inflammatory signaling and dysfunction of osteoarthritic chondrocytes. Int. J. Mol. Med., 35 (2015), pp. 1657-1666

[18] R.C. Team. R: a Language and Environment for Statistical Computing. R Foundation for Statistical Computing, Vienna, Austria (2012). http://wwwr-projectorg/

[19] V. Gkretsi, T. Simopoulou, A. Tsezou. Lipid metabolism and osteoarthritis: lessons from atherosclerosis. Prog. Lipid Res., 50 (2011), pp. 133-140

[20] Q. Zhuo, W. Yang, J. Chen, Y. Wang. Metabolic syndrome meets osteoarthritis. Nat. Rev. Rheumatol., 8 (2012), pp. 729-737

[21] M.D. Tortorella, A.M. Malfait, C. Deccico, E. Arner. The role of ADAM-TS4 (aggrecanase-1) and ADAM-TS5 (aggrecanase-2) in a model of cartilage degradation. Osteoarthr. Cartil., 9 (2001), pp. 539-552

[22] E.C. Arner. Aggrecanase-mediated cartilage degradation. Curr. Opin. Pharm., 2 (2002), pp. 322-329 
[23] B.C. Sondergaard, K. Henriksen, H. Wulf, S. Oestergaard, U. Schurigt, R. Brauer, et al. Relative contribution of matrix metalloprotease and cysteine protease activities to cytokine-stimulated articular cartilage degradation. Osteoarthr. Cartil., 14 (2006), pp. 738-748

[24] M.A. Karsdal, E.U. Sumer, H. Wulf, S.H. Madsen, C. Christiansen, A.J. Fosang, et al. Induction of increased cAMP levels in articular chondrocytes blocks matrix metalloproteinase-mediated cartilage degradation, but not aggrecanase-mediated cartilage degradation. Arthritis Rheum., 56 (2007), pp. 1549-1558

[25] J.B.J. van Meurs, P. van Lent, A.E.M. Holthuysen, I.I. Singer, E.K. Bayne, W.B. van den Berg. Kinetics of aggrecanase- and metalloproteinase-induced neoepitopes in various stages of cartilage destruction in murine arthritis. Arthritis Rheum., 42 (1999), pp. 1128-1139

[26] B. Andruski, D.M. McCafferty, T. Ignacy, B. Millen, J.J. McDougall. Leukocyte trafficking and pain behavioral responses to a hydrogen sulfide donor in acute monoarthritis. Am. J. Physiol. Regul. Integr. Comp. Physiol., 295 (2008), pp. R814R820

[27] E. Ekundi-Valentim, K. Santos, E. Camargo, A. Denadai-Souza, S. Teixeira, C.I. Zanoni, et al. Differing effects of exogenous and endogenous hydrogen sulphide in carrageenan-induced knee joint synovitis in the rat. Br. J. Pharmacol., 159 (2010), pp. $1463-1474$

[28] W.P. Chen, J.L. Tang, J.P. Bao, P.F. Hu, C. Yu, Z.L. Shi, et al .Effects of diallyl sulphide in chondrocyte and cartilage in experimental osteoarthritis in rabbit. Phytother. Res., 25 (2011), pp. 351-356

[29] G.A. Benavides, G.L. Squadrito, R.W. Mills, H.D. Patel, T.S. Isbell, R.P. Patel, et al .Hydrogen sulfide mediates the vasoactivity of garlic. Proc. Natl. Acad. Sci. U. S. A., 104 (2007), pp. 17977-17982

[30] S. Zhang, C. Pan, F. Zhou, Z. Yuan, H. Wang, W. Cui, et al. Hydrogen sulfide as a potential therapeutic target in fibrosis. Oxid. Med. Cell Longev., 2015 (2015), p. 12. Article ID 593407. http://dx.doi.org/10.1155/2015/593407

[31] T.M. Simon, D.W. Jackson. Articular cartilage: injury pathways and treatment options. Sports Med. Arth. Rev., 14 (2006), pp. 146-154

[32] L. Chen, S. Ingrid, Y. Ding, Y. Liu, J. Qi, C. Tang, et al. Imbalance of endogenous homocysteine and hydrogen sulfide metabolic pathway in essential hypertensive children. Chin. Med. J., 120 (2007), pp. 389-393

[33] M. Whiteman, K. Gooding, J. Whatmore, C.I. Ball, D. Mawson, K. Skinner, et al. Adiposity is a major determinant of plasma levels of the novel vasodilator hydrogen sulphide. Diabetologia, 53 (2010), pp. 1722-1726

[34] Y.H. Chen, W.Z. Yao, B. Geng, Y.L. Ding, M. Lu, M.W. Zhao, et al. Endogenous hydrogen sulfide in patients with COPD. Chest, 128 (2005), pp. 3205-3211

[35] A.A. Vela-Anero, L. Gato-Calvo, C. Ruiz-Romero, R. Meijide-Failde, F.J. Blanco, E.F. Burguera. Endogenous hydrogen sulfide production is reduced in OA cartilage. Possible contribution to the pathogenesis of OA. Osteoarthr. Cartil., 23 (2015), p. A311

[36] G. Sherman, L. Zeller, A. Avriel, M. Friger, M. Harari, S. Sukenik. Intermittent balneotherapy at the Dead Sea area for patients with knee osteoarthritis. Isr. Med. Assoc. J., 11 (2009), pp. 88-93

[37] C. Kovacs, A. Bozsik, M. Pecze, I. Borbely, A. Fogarasi, L. Kovacs, et al. Effects of sulfur bath on hip osteoarthritis: a randomized, controlled, single-blind, follow-up trial: a pilot study. Int. J. Biometeorol., 60 (2016), pp. 1675-1680

[38] R. Forestier, F.B. Erol Forestier, A. Francon. Spa therapy and knee osteoarthritis: a systematic review. Ann. Phys. Rehab. Med., 59 (2016), pp. 216-226

[39] Fioravanti, L. Cantarini, G.M. Guidelli, M. Galeazzi. Mechanisms of action of spa therapies in rheumatic diseases: what scientific evidence is there?. Rheumatol. Int., 31 (2011), pp. 1-8 